OPEN ACCESS

Edited by:

Jason Sahl,

Northern Arizona University, United States

Reviewed by:

Ulises Garza-Ramos,

National Institute of Public Health,

Mexico

Balaji Veeraraghavan Christian Medical College \& Hospital,

India

*Correspondence:

Tatiana Amabile de Campos tatianadecampos@unb.br

Specialty section:

This article was submitted to

Antimicrobials, Resistance

and Chemotherapy,

a section of the journal

Frontiers in Microbiology

Received: 08 September 2020

Accepted: 25 March 2021

Published: 16 April 2021

Citation:

Campos TA, Almeida FM Almeida APC, Nakamura-Silva $R$

Oliveira-Silva M, Sousa IFA, Cerdeira L, Lincopan N, Pappas GJ Jr

and Pitondo-Silva A (2021)

Multidrug-Resistant (MDR) Klebsiella variicola Strains Isolated in a Brazilian

Hospital Belong to New Clones.

Front. Microbiol. 12:604031.

doi: 10.3389/fmicb.2021.604031

\section{Multidrug-Resistant (MDR) Klebsiella variicola Strains Isolated in a Brazilian Hospital Belong to New Clones}

\author{
Tatiana Amabile de Campos ${ }^{1,2 *}$, Felipe Marques de Almeida ${ }^{3}$, \\ Ana Paula Cardoso de Almeida ${ }^{2}$, Rafael Nakamura-Silva ${ }^{4}$, Mariana Oliveira-Silva ${ }^{4}$, \\ Isabela Felix Alencar de Sousa ${ }^{3}$, Louise Cerdeira ${ }^{5,6}$, Nilton Lincopan $^{6}$, \\ Georgios Joannis Pappas Jr. ${ }^{1,3}$ and André Pitondo-Silva ${ }^{4,7}$
}

\begin{abstract}
1 Departamento de Biologia Celular, Instituto de Ciências Biológicas, Universidade de Brasilia, Brasilia, Brazil, ${ }^{2}$ Programa de Pós-graduação em Biologia Microbiana, Universidade de Brasilia, Brasília, Brazil, ${ }^{3}$ Programa de Pós-graduação em Biologia Molecular, Universidade de Brasília, Brasilia, Brazil, ${ }^{4}$ Programa de Pós-graduação em Tecnologia Ambiental, Universidade de Ribeirão Preto, Ribeirão Preto, Brazil, ${ }^{5}$ Instituto de Ciências Biológicas, Universidade de São Paulo, São Paulo, Brazil, ${ }^{6}$ Department of Infectious Diseases, Central Clinical School, Monash University, Melbourne, VIC, Australia, ${ }^{7}$ Programa de Pós-graduação em Odontologia, Universidade de Ribeirão Preto, Ribeirão Preto, Brazil
\end{abstract}

Klebsiella variicola is mainly associated with opportunistic infections and frequently identified as Klebsiella pneumoniae. This misidentification implies a wrong epidemiology result as well as incorrect attribution to $K$. pneumoniae as the etiology of some severe infections. Recently, huge efforts have been made to study $K$. variicola, however, the biological aspects of this species are still unclear. Here we characterized five K. variicola strains initially identified as $K$. pneumoniae, with a Vitek-2 System and $16 S$ rRNA sequencing. One-step multiplex polymerase chain reaction and Whole Genome Sequencing (WGS) identified them as K. variicola. Additionally, WGS analysis showed that all the strains are closely related with $K$. variicola genomes, forming a clustered group, apart from K. pneumoniae and K. quasipneumoniae. Multilocus sequence typing analysis showed four different sequence types (STs) among the strains and for two of them (Kv97 and Kv104) the same ST was assigned. All strains were multidrug-resistant (MDR) and three showed virulence phenotypes including invasion capacity to epithelial cells, and survival in human blood and serum. These results showed the emergence of new $K$. variicola clones with pathogenic potential to colonize and cause infection in different tissues. These characteristics associated with MDR strains raise great concern for human health.

Keywords: Klebsiella variicola, antimicrobial resistance, whole genome sequence, pathogenicity, brazilian clones, multidrug-resistant

\section{INTRODUCTION}

Klebsiella variicola is a Gram-negative, facultative anaerobic and non-motile bacillus belonging to Enterobacterales. The species is mainly associated with opportunistic infections, such as those of the bloodstream (BSI) (Maatallah et al., 2014), urinary tract (Potter et al., 2018), respiratory tract (Rodríguez-Medina et al., 2019), and neonatal outbreaks (Piepenbrock et al., 2020). 
When associated with BSI, the infections presented high mortality rates, but the strains did not show known virulence factors such as capsular types or mucoid phenotype (Maatallah et al., 2014). Although it has an opportunistic nature, hypervirulent strains were described in outbreaks in Bangladesh (Farzana et al., 2019) and China (Lu et al., 2018). The association among hypervirulence and multidrug resistance becomes a more worrying scenario for the public healthcare system.

$K$. variicola are frequently misidentified as $K$. pneumoniae by conventional biochemical tests and automated microbiology systems used worldwide (Rodríguez-Medina et al., 2019). Nevertheless, genomic information surveys have recently demonstrated that this species is also associated with severe human infections (Fonseca et al., 2017; Barrios-Camacho et al., 2019; Srinivasan and Rajamohan, 2020). Although not widely studied as $K$. pneumoniae, there are reports indicating that $K$. variicola shows clear differences related to infection, highlighting the importance of its early diagnosis (RodríguezMedina et al., 2019). In this report we characterized five K. variicola strains (Kv15, Kv35, Kv57, Kv97, and Kv104), first identified as $K$. pnenumoniae. All these strains were submitted to genomic characterization to determine their sequence types (STs), virulence, and antimicrobial resistance (AMR) genes. Biological assays such as toxicity and invasion to epithelial cells, hypermucosviscousity and survival in human blood and serum were performed to evaluate their pathogenic potential.

\section{MATERIALS AND METHODS}

\section{Bacterial Strains Isolation, Growth Conditions, and Storage}

The five $K$. variicola strains included in the present study, named Kv15, Kv35, Kv57, Kv97, and Kv104, were isolated at the University Hospital of Brasília, the tertiary hospital at the University of Brasília, located in Brasília in the Brazilian Federal District (DF), between September 2013 and December 2017. The strains were initially identified as K. pneumoniae by a VITEK 2 system (BioMérieux) and maintained at $-80^{\circ} \mathrm{C}$ in $15 \%$ glycerol.

Thereafter, the bacterial identification was confirmed by molecular methods. Initially, the isolates were typed by $16 \mathrm{~S}$ rRNA sequencing (Weisburg et al., 1991). After that, K. variicola identification was confirmed by means of the one-step multiplex polymerase chain reaction (PCR) protocol proposed by Fonseca et al. (2017). All K. variicola identified were used for biological and genomic characterization.

\section{Antimicrobial Susceptibility Profile}

Antimicrobial susceptibility testing was performed by the disk diffusion method as recommended by the Clinical and Laboratory Standards Institute (CLSI) (2020). All antibiotics recommended for Enterobacterales were tested, considering the particularities for Klebsiella spp. For this approach 37 different antibiotic disks (Oxoid Ltd., Basingstoke, United Kingdom) were used (Supplementary Material 1). Each strain was considered susceptible or non-susceptible (either intermediate or resistant) to each antibiotic tested. Based on the susceptibility profile, each strain was classified into different categories including, multidrug-resistant (MDR), extensively drug-resistant (XDR) and pandrug-resistant (PDR) (Magiorakos et al., 2012).

\section{Biofilm Production Assays}

A volume of $100 \mu \mathrm{L}$ from an optical density at $600 \mathrm{~nm}\left(\mathrm{OD}_{600}\right.$ $n m$ ) culture of each strain, previously grown in Luria-Bertani (LB) broth (Sigma Aldrich) at $37^{\circ} \mathrm{C}$, were transferred to a well of a 24 well micro-plate containing $100 \mu \mathrm{L}$ of LB. After $24 \mathrm{~h}$ of incubation at $37^{\circ} \mathrm{C}$, the supernatant was discharged and the plates were washed twice with $\mathrm{NaCl} 0.9 \%$. The pellets were fixed with methanol (15 $\mathrm{min}$ at room temperature), dried, and stained with violet crystal. After the solubilization with acetic acid 33\%, the $\mathrm{OD}_{570} \mathrm{~nm}$ absorbance from each well was read using a Spectramax (Promega). The assay was performed in triplicate for all strains and for the negative control (Stepanović et al., 2004).

\section{Hypermucosviscosity}

The hypermucoviscous (Hmv) phenotype was investigated by a string test (Mazloum et al., 2016). The strains were inoculated on Mueller-Hinton agar (Oxoid) and incubated for $24 \mathrm{~h}$ at $37^{\circ} \mathrm{C}$. Following bacterial growth, a bacteriological loop was used to touch and lift vertically an isolated colony. Formation of a mucus filament of $>5 \mathrm{~mm}$ was considered positive for hypermucoviscosity. All the strains were tested in triplicate.

\section{Bacterial Human Blood and Serum Survival}

Bacterial suspensions $\left(10^{7}\right.$ bacterial cells) obtained from a culture of $18 \mathrm{~h}$ grown at $37^{\circ} \mathrm{C}$ in $\mathrm{LB}$ broth were added in $550 \mu \mathrm{L}$ of human blood and in $550 \mu \mathrm{L}$ human serum and incubated for 0 , 30,60 , and $120 \mathrm{~min}$ at $37^{\circ} \mathrm{C}$. Thereafter, aliquots of $10 \mu \mathrm{L}$ from each inoculum were plated in MacConkey Agar (Oxoid Ltd.) and the plates were incubated at $37^{\circ} \mathrm{C}$ for $18 \mathrm{~h}$ for colony forming unit (CFU) recovery quantification (DeLeo et al., 2017). For the assays, blood and serum were collected from five healthy donors. All strains were tested in triplicate for each donor.

\section{Bacterial Invasion and Toxicity to HEp-2 Epithelial Cells}

Bacterial invasion to epithelial cells and cytotoxicity were evaluated with HEp-2 cells by using multiplicity of infection 1:1,000 (HEp-2 cells: bacterial strains) (Favre-Bonte et al., 1999). Following the infection, the HEp-2 cells were incubated at $37^{\circ} \mathrm{C}$ and $5 \% \mathrm{CO}_{2}$. After 30 min the cells were washed with phosphatebuffered saline (PBS) and the medium was replaced by Dulbecco's Modified Eagle's medium (Gibco) with $10 \mathrm{mg} / \mathrm{mL}$ of gentamicin (Sigma Aldrich). Bacterial invasion and intracellular survival were evaluated by CFU quantification at $24 \mathrm{~h}$ post infection (h.p.i.). Bacterial cytotoxicity was determined by MTT (3-4, 5-dimethylthiazolyl-2)-2, 5-diphenyltetrazolium bromide assays (Sigma Aldrich) at 3 h.p.i (Campos et al., 2018). All assays were carried out in triplicate for all strains. 


\section{Genome Sequencing}

Genomic DNA samples were sequenced on an Illumina MiSeq platform (Illumina Inc., San Diego, CA) using 250-bp paired-end reads for the strains Kv15, Kv97, and Kv104 and on an Illumina NextSeq platform (Illumina Inc., San Diego, CA), using 75-bp paired-end reads for the strains Kv35 and Kv57.

\section{Genome Assembly and Annotation}

De novo genome assembly was performed with Unicycler (Wick et al., 2017) v0.4.8 using the MpGAP pipeline v1.0 with default parameters. Genome completeness was assessed with BUSCO v4.1.0, using the enterobacterales_odb10 dataset (Seppey et al., 2019).

Genome annotation was performed with the bacannot pipeline v1. $0^{2}$ using $85 \%$ gene coverage and identity thresholds for virulence gene annotations. Through this pipeline, AMR genes were detected with CARD-RGI database (version 3.0.7) (Jia et al., 2017) and AMRFinderPlus v3.2.1 (Feldgarden et al., 2019) tools, while VFDB (Liu et al., 2019) was used to identify virulence factors (accessed in November 2019). Additionally, AMR genes were also predicted using ResFinder 4.0 (Bortolaia et al., 2020). Capsule synthesis (K) and lipopolysaccharide (O) loci were identified using Kaptive (Wick et al., 2018). Multilocus sequence typing (MLST) was performed with $K$. variicola MLST scheme (accessed on 1th September 2019) (Barrios-Camacho et al., 2019). Plasmid replicons were detected with Plasmidfinder v2.1 (Carattoli et al., 2014).

\section{Comparative Genomics}

Genome-based taxonomy analysis was performed with the Type Strain Genome Server (TYGS) (Meier-Kolthoff and Göker, 2019). Average Nucleotide Identity among the five genomes were calculated with fastANI v1.3 (Jain et al., 2018b) and whole genome homology maps were produced with Mashmap (Jain et al., 2018a). The genomes of the five strains were aligned with snippy v4.6.0 $0^{3}$, using default parameters, to detect single nucleotide polymorphism among them.

For species-level comparative analysis, all the Klebsiella variicola complete genomes available in NCBI Refseq in December 2019 were downloaded via ncbi-genome-download tool $^{4}$, totalizing 296 genomes.

With these genomes, an alignment-free genomic distance matrix among them was constructed with Dashing v0.5-7g841b (Baker and Langmead, 2019) with default parameters. The estimated genomic distances generated by Dashing were in turn projected into a two-dimensional space using multidimensional scaling analysis (MDS), by means of custommade R language scripts. K. variicola pangenome was estimated using PPanGGOLIN v1.1.72 (Gautreau et al., 2020) with default parameters. This pangenome was further used to align and create

\footnotetext{
${ }^{1}$ https://github.com/fmalmeida/MpGAP

${ }^{2}$ https://github.com/fmalmeida/bacannot

${ }^{3}$ https://github.com/tseemann/snippy

${ }^{4}$ https://github.com/kblin/ncbi-genome-download
}

a gene presence/absence matrix of genes of interest (such as the presence of virulence genes) using PPanGGOLiN align module.

All the MLST profiles deposited in the $K$. variicola MLST database $^{5}$ were downloaded in October 2020 and were used to reconstruct the genetic relationships among them using a minimum spanning tree algorithm (MSTree V2) implemented in GrapeTree v2.2 (Zhou et al., 2018).

\section{Statistical Analysis}

Statistical analysis was performed by using GraphPadPrism v. 8.0 (GraphPad Software, San Diego California United States). ANOVA one-way was used to determine the bacterial invasion capacities and intracellular survival. The $t$-test was used for bacterial toxicity for HEp-2 cells determination.

\section{RESULTS}

\section{Bacterial Strains Isolation and Identification}

The strains studied in this work were obtained from a K. pneumoniae collection containing 104 isolates from different patients and sources from September 2013 to January 2017. Among all isolates, five were later identified as K. variicola (Table 1), corresponding to $4.8 \%$ of prevalence within this allegedly $K$. pneumoniae collection. For $K$ variicola identification, we used three different techniques: Vitek2 (BioMérieux), 16S rRNA sequencing, and one-step multiplex PCR protocol. Vitek2 identified the strains as K. pneumoniae, whereas the $16 \mathrm{~S}$ sequencing showed $100 \%$ identity to both $K$. variicola and $K$. pneumoniae. Conversely, the multiplex PCR approach identified all the strains as K. variicola, showing its adequacy to differentiate the two species in this context.

\section{Antimicrobial Susceptibility and Biological Assays}

Disk diffusion test showed that all strains were classified as multidrug resistant (MDR), as they were non-susceptible to three different antimicrobial classes (Table $\mathbf{1}$ and Supplementary Material 1). In vitro assays were performed to determine hypermucoviscous phenotype, biofilm production, serum and blood bacterial survival, epithelial cells invasion and citoxicity (Table 1 and Figure 1). Overall, the strains presented capacity to survive and grow after $60 \mathrm{~min}$ of incubation in human blood and serum (Figures 1A,B). The strain Kv57, isolated from a catheter culture of a 55 year old man, did not show Hmv phenotype, but presented survival and growth in human serum, as well as high invasion capacity in epithelial cells and high cytotoxicity to HEp-2 cells (reducing $75 \%$ of cell viability) (Table 1 and Figure 1). Kv97 and Kv104, isolated from urine culture from elderly female patients, presented Hmv phenotype and strong biofilm production (Table 1). However, both strains showed

${ }^{5} \mathrm{http}: / / \mathrm{mlstkv} \cdot \mathrm{insp} \cdot \mathrm{mx} /$ 
TABLE 1 | Isolation data, biofilm production, hypermucousviscous phenotype and antimicrobial resistance profile of Klebsiella variicola strains.

\begin{tabular}{|c|c|c|c|c|c|c|c|}
\hline \multirow[t]{2}{*}{ Strain ID } & \multirow[t]{2}{*}{ Culture } & \multicolumn{2}{|c|}{ Patient data } & \multirow{2}{*}{$\begin{array}{l}\text { Isolation period } \\
\text { (year-month) }\end{array}$} & \multirow[t]{2}{*}{ Hmv } & \multirow{2}{*}{$\begin{array}{c}\text { Biofilm } \\
\text { production }\end{array}$} & \multirow[t]{2}{*}{ Antimicrobial resistance profile* } \\
\hline & & Genera & $\begin{array}{c}\text { Birth date } \\
\text { (year-month) }\end{array}$ & & & & \\
\hline Kv15 & Urine & $\mathrm{F}$ & 1942/Aug & 2015/Apr & - & STR & $\begin{array}{l}\text { MDR } \\
\text { (tobramycin, doxycycline, nitrofurantoin, } \\
\text { tetracycline, sulfonamide, } \\
\text { trimethoprim + sulfamethoxazole, } \\
\text { trimethoprim, nalidixic acid) }\end{array}$ \\
\hline Kv35 & Blood & $\mathrm{F}$ & 1973/Sept & 2015/Sept & - & W & $\begin{array}{l}\text { MDR } \\
\text { (ciprofloxacin, tobramycin, cefuroxime, } \\
\text { nitrofurantoin, sulphonamide, } \\
\text { trimethoprim + sulfamethoxazole, } \\
\text { trimethoprim, } \\
\text { nalidixic acid) }\end{array}$ \\
\hline Kv57 & Catheter & M & 1961/Sept & 2016/Mar & - & M & $\begin{array}{l}\text { MDR } \\
\text { (tobramycin, doxycycline, nitrofurantoin, } \\
\text { sulphonamide, } \\
\text { trimethoprim + sulfamethoxazole, } \\
\text { trimethoprim) }\end{array}$ \\
\hline Kv97 & Urine & $\mathrm{F}$ & 1940/Mar & 2017/Jan & + & STR & $\begin{array}{l}\text { MDR } \\
\text { (ciprofloxacin, tobramycin, nitrofurantoin, } \\
\text { sulphonamide, } \\
\text { trimethoprim + sulfamethoxazole, } \\
\text { trimethoprim) }\end{array}$ \\
\hline Kv104 & Blood & $\mathrm{F}$ & 1959/Aug & 2017/Jan & + & STR & $\begin{array}{l}\text { MDR } \\
\text { (ciprofloxacin, tobramycin, nitrofurantoin, } \\
\text { sulphonamide, } \\
\text { trimethoprim + sulfamethoxazole, } \\
\text { trimethoprim) }\end{array}$ \\
\hline
\end{tabular}

F, Female; M, Male; Mar, Mach; Aug, August; Sept, September; Hmv, hypermucousviscous phenotype; ST, sequence type; STR, Strong biofilm production M, Moderate

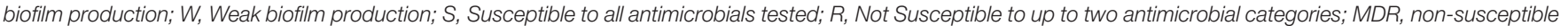
to one or more agents in more the three antimicrobial categories; * all strains are intrinsically resistant to ampicillin.

A

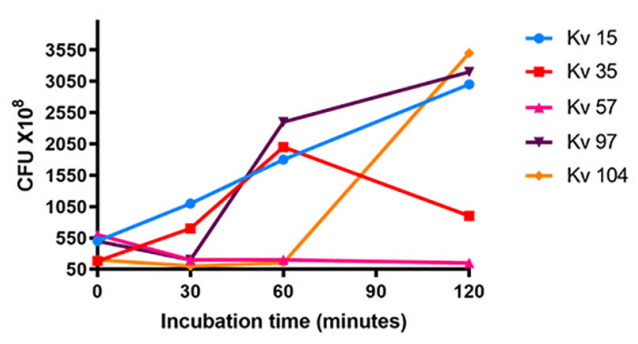

C

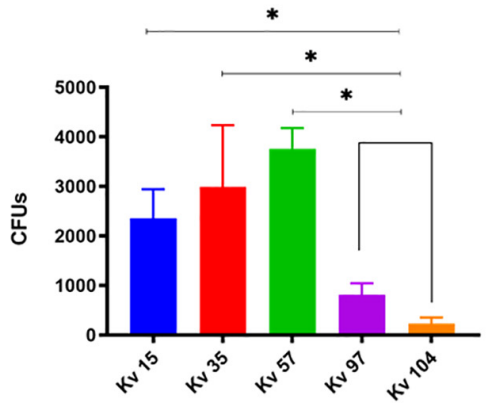

B

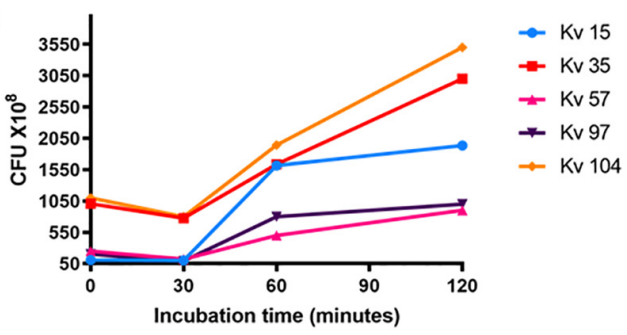

D

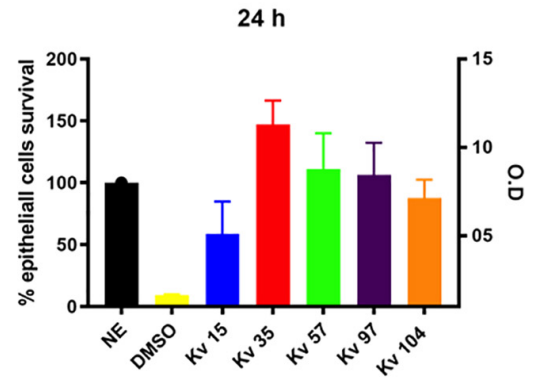

FIGURE 1 | Biological assays used to determine the pathogenic potential of Klebsiella variicola strains. (A) Bacterial survival after incubation in human blood for different times at $37^{\circ} \mathrm{C}$. (B) Bacterial survival after incubation in human serum for different times at $37^{\circ} \mathrm{C}$. (C) Bacterial invasion in HEp-2 cells. (D) Bacterial cytotoxicity to HEp-2 cells. The bacterial survival was determined by CFU counting of strains plated from the incubation in blood and serum. Bacterial invasion was determined by CFU counting of strains plated after HEp-2 lysis. Cytotoxicity to HEp-2 cells were determined by the Griss Method. All assays were performed in triplicate. ANOVA one-way were used for statical analysis, ${ }^{*} p<0.05$ values were considered significant. 
low capacity to invade Hep2 cells when compared to the others (Figure 1C).

\section{Genomic Analyses}

Sequence data obtained by short-read DNA sequencing was used to assemble the genomes of the five studied strains. The resulting genome assemblies averaged $5.5 \mathrm{Mb}$ and showed high levels (>95\%) of gene space completeness based on BUSCO metrics (Supplementary Table 1). Aside from the chromosomal scaffold, the $\operatorname{IncFIB}(\mathrm{K})$ plasmid replicon was identified in the genomes of Kv35, Kv97, and Kv104 (Supplementary Table 1). All the genomes have been classified as K. variicola by the Type Strain Genome Server (TYGS).

\section{Molecular Typing}

The identification of $\mathrm{K}$ and $\mathrm{O}$ serotypes is important for rapid detection of outbreaks or high-risk clones, given their links to increased virulence, as is the case of $\mathrm{K} 1$ and $\mathrm{O} 1$ serotypes (Hsieh et al., 2012; Fang et al., 2016; Patro and Rathinavelan, 2019). Genome analysis enabled the detection of four different capsular synthesis loci (KL60, KL71, KL111, and KL20) and one lipopolysaccharide locus shared among the genomes (O3) (Table 2). In K. pneumoniae, the $\mathrm{O} 3$ strains is one of the most common serotypes, after $\mathrm{O} 1$ and $\mathrm{O} 2$.

Using the INSP MLST scheme for K. variicola, four new STs were identified (ST168, ST170, ST171, and ST172), with Kv97 and Kv104 assigned as ST172 (Table 2). The genetic relationships among all known $K$. variicola STs was visualized with GrapeTree, which indicated that the species can be divided into at least two clonal groups (CGs) that seem to originate from STs 19 and 54 (Figure 2). This analysis shows that the studied strains were allocated in both CGs. Interestingly, the strains Kv97 and Kv104, which are the less invasive to Hep-2 cells (Figure 1C and Table 1) have been placed in the CG with ST19 (Figure 2) as a founder whereas the other strains (Kv15, Kv35, and Kv57) were grouped in the other CG with ST54 as a founder (Figure 2). This observation suggests that these CGs may represent populations of $K$. variicola with different phenotypes and gene content dynamics similar to K. pneumoniae (Wyres et al., 2019, 2020). Moreover, all the strains from Brazil, including the strains of this study, have been spread in different branches of the tree, highlighting the clonal diversity in Brazil that still needs to be measured.

\section{Antimicrobial Resistance (AMR) Genes and Virulence Genes (VG)}

To support the experimental observation that all analyzed strains are classified as MDR (Table 1), genomic sequences were used to search for AMR genes using three software tools and databases, namely AMRFinderPlus, CARD and Resfinder. The combined results showed the presence of genes related to intrinsic betalactam resistance $\left(a m p \mathrm{H}, b l a_{\mathrm{LEN}-2}, b l a_{\mathrm{LEN}-9}, b l a_{\mathrm{LEN}-12}\right)$ and fosfomycin (fos A) in all strains (Table 2). Moreover, the efflux pump oq $x A B$ was also found and has been implicated in low to intermediate resistance to quinoxalines, quinolones, tigecycline, nitrofurantoin, several detergents and disinfectants. In addition, Resfinder identified mutations in the genes acr R, ompK36, and ompK37, that can possibly confer resistance to fluoroquinolones, cephalosporins, and carbapenem, however, further investigation of these mutations is still necessary. The efflux pump acrAB was also found among all strains. When overexpressed, this efflux pump contributes to multidrug resistance. Besides, acr AB is a resistance factor widespread in K. pneumoniae and can to contribute to virulence by providing resistance to host-derived antimicrobial peptides (Swick et al., 2011; Table 2).

TABLE 2 | Antimicrobial resistance genes (ARG), virulence genes (VG), and molecular typing of Klebsiella variicola strains.

\begin{tabular}{|c|c|c|c|c|c|}
\hline Strain ID & ARG & $\mathbf{K}$ & 0 & VG & ST \\
\hline Kv15 & $\begin{array}{c}\text { oqxAB, fosA, } \\
\text { bla }{ }_{\text {LEN-9 }}, \text { ampH, } \\
\text { acrAB }\end{array}$ & KL60 & O3 & $\begin{array}{l}\text { Capsule, enterobactin, } \\
\text { type } 1 \text { fimbriae, type3 } \\
\text { fimbriae, acrR, } \\
\text { ompK36, ompK37 }\end{array}$ & 170 \\
\hline Kv35 & $\begin{array}{c}\text { oqxAB, fosA, } \\
\text { bla } \\
\text { ack }-2, a m p H \\
\text { acrAB }\end{array}$ & $\mathrm{KL} 71$ & O3 & $\begin{array}{l}\text { Capsule, enterobactin, } \\
\text { Type } 1 \text { fimbriae, Type3 } \\
\text { fimbriae, acrR, } \\
\text { ompK36, ompK37 }\end{array}$ & 171 \\
\hline Kv57 & $\begin{array}{c}\text { oqxAB, fosA, } \\
\text { bla } 2 \text { LEN-2, ampH, } \\
\text { acrAB }\end{array}$ & KL111 & O3 & $\begin{array}{l}\text { Capsule, enterobactin, } \\
\text { type } 1 \text { fimbriae, type3 } \\
\text { fimbriae, acrR, } \\
\text { ompK36, ompK37 }\end{array}$ & 168 \\
\hline Kv97 & $\begin{array}{c}\text { oqxAB, fosA, } \\
\text { bla } 2 \text { LEN12, ampH, } \\
\text { acrAB }\end{array}$ & KL2O & $\mathrm{O} 3$ & $\begin{array}{l}\text { Capsule, enterobactin, } \\
\text { type } 1 \text { fimbriae, type3 } \\
\text { fimbriae, acrR, } \\
\text { ompK36, ompK37 }\end{array}$ & 172 \\
\hline Kv104 & $\begin{array}{c}\text { oqxAB, fosA, } \\
\text { bla } \\
\text { LEN } 12, \text { ampH, } \\
\text { acrAB }\end{array}$ & KL20 & O3 & $\begin{array}{l}\text { Capsule, enterobactin, } \\
\text { type } 1 \text { fimbriae, typ3 } \\
\text { fimbriae, acrR, } \\
\text { ompK36, ompK37 }\end{array}$ & 172 \\
\hline
\end{tabular}

K, capsular antigen type; O, O antigen type; ST, sequence type. 


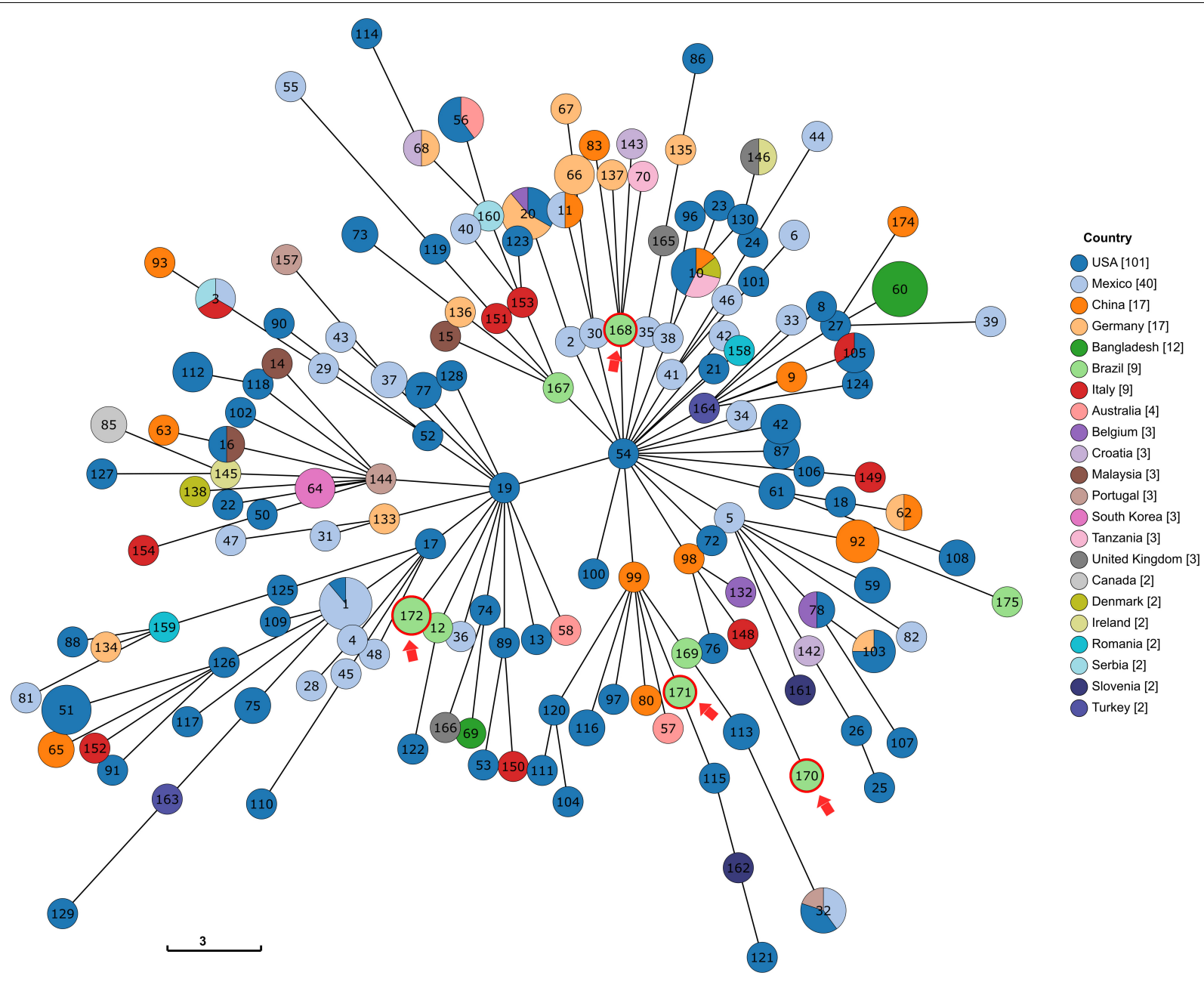

FIGURE 2 | Tree representation of the genetic relationships among the different profiles of the K. variicola MLST scheme, produced via GrapeTree with the minimum spanning tree algorithm (MSTree V2). The STs identified in this study are shown with a red arrow. For readability purposes, all the genomes that were the only representant of a country were removed.

The search for virulence genes identified that the strains harbored classical $K$. pneumoniae virulence factors related to the phenolate siderophore enterobactin, type 1 fimbriae, and the E. coli common pilus (ECP) locus (Table 2), an adhesive structure produced by all E. coli pathogroups that is also produced by Klebsiella pneumoniae strains (Alcántar-Curiel et al., 2013). Type 3 fimbriae $m r k \mathrm{ABCDF}$ and $m r k \mathrm{HI}$ gene loci, which mediate bacterial adhesion and biofilm formation in several surface structures (Wilksch et al., 2011), were also detected in the genomes. Moreover, all strains harbored multiple genes from the type VI secretion system (T6SS), an apparatus involved in bacterial competition, cell invasion, and in vivo colonization (Ho et al., 2014; Barbosa and Lery, 2019).

For a global perspective, the presence/absence of identified virulence genes was contrasted against several available K. variicola genomes. The results show that, apart from genes related to T6SS, the majority of these virulence genes are widespread in the species (Figure 3). The differences observed in this analysis are not sufficient to explain the different phenotypes observed. Moreover, this comparative analysis suggests that these classical $K$. pneumoniae genes are also very conserved in $K$. variicola, indicating that the species may have other genes that contribute to the virulence phenotype as also suggested in previous studies (Potter et al., 2018; Rodríguez-Medina et al., 2019).

\section{Comparative Genomics}

Genome similarity among the five genomes was investigated by calculating their Average Nucleotide Identity (ANI) with fastANI. Aside from an almost identical ANI value (99.99) between strains $\mathrm{Kv} 97$ and $\mathrm{Kv} 104$, the analysis reveals a minimum ANI of 98.88 between strains Kv35 and Kv104 and a maximum of 99.16 between the strains Kv15 and Kv57. Whole genome homology maps among the five genomes were constructed with Mashmap (Supplementary Figure 1). The resulting dotplots indicate that some of the strains might have regions of duplication (Supplementary Figures 1C,D) but, in general, it shows an overall collinearity between the genomes (Supplementary Figure 1). The homology map between the strains Kv97 and Kv104 shows that their genomes are virtually 


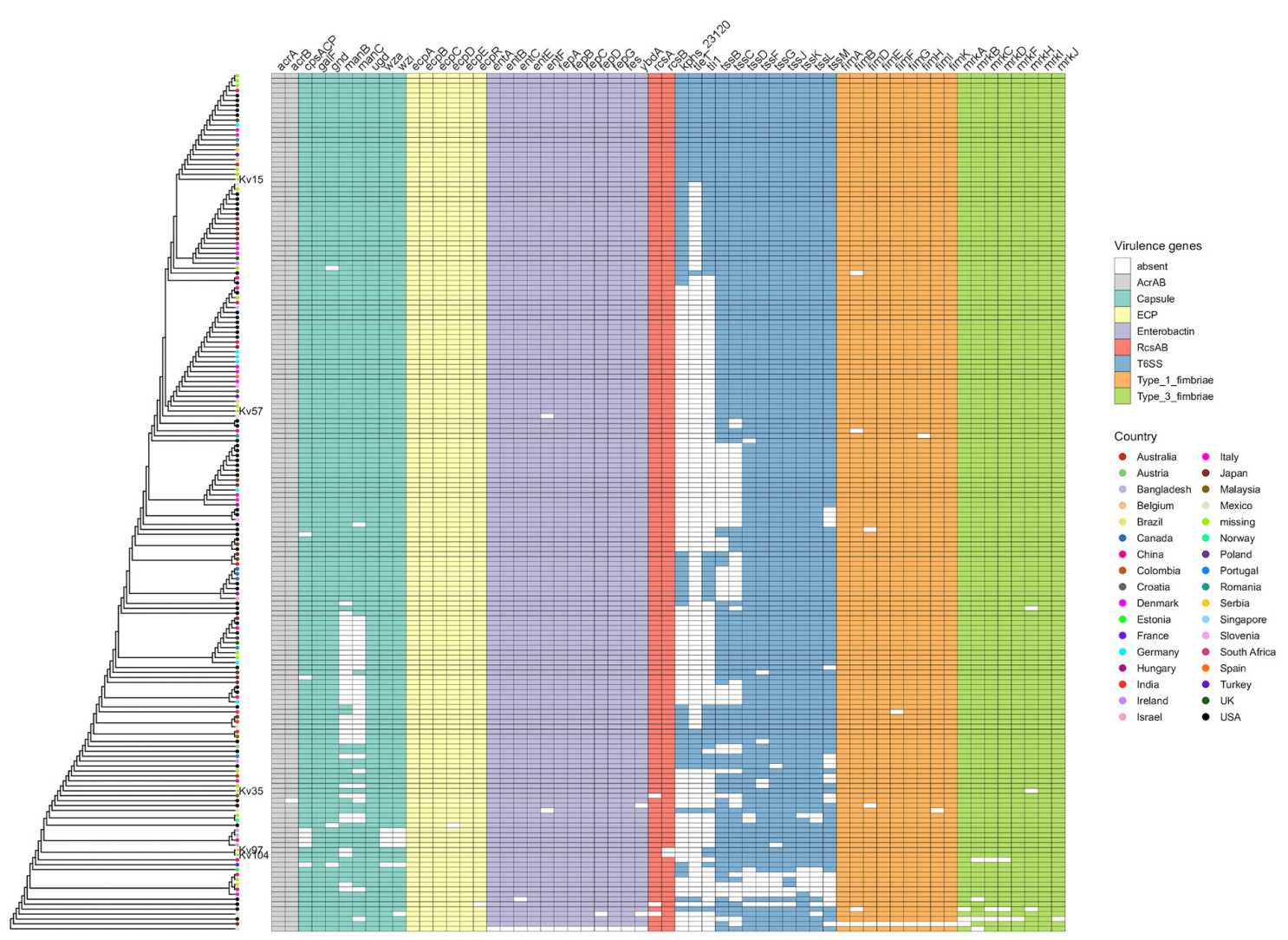

FIGURE 3 | Distribution of $K$. pneumoniae virulence factors among $K$. variicola genomes, represented in a presence/absence matrix of genes. The genomes have been clustered based on their gene content.

identical, supporting their high ANI value (Supplementary Figure 1A). These results indicate that the strains Kv97 and Kv104 are clonal, further supported by the inference of only 19 SNPs computed from their whole genome alignment. We extended the comparative genomics analysis by including 296 K. variicola complete genomes available in NCBI's Refseq and creating an all-vs.-all distance matrix based on Dashing software metrics. The projection of this matrix into a two-dimensional space using multidimensional scaling analysis (MDS) enabled the visualization of the relationships of all the genomes (Supplementary Figure 2). This analysis shows that, although isolated from the same hospital, these genomes do not cluster together and individually share genetic relationships to different isolates from the global collection, with the closest genomes to the five strains originating from different countries including the United States, Belgium and China (Table 3). Altogether, this suggests multiple introduction routes and dispersal potential of K. variicola.

\section{DISCUSSION}

Currently, the misidentification of K. variicola as K. pneumoniae in routine diagnostics tests is observed. Thus, many worldwide epidemiological data on infections caused by $K$. variicola may have been wrongly attributed to K. pneumoniae, underscoring the urge to better characterize this species regarding its genetics, pathogenesis, and ecology, given its ubiquitous distribution (Rodríguez-Medina et al., 2019). In particular, knowledge regarding the genome content of $K$. variicola strains is important to provide insights regarding the plasticity of this species to acquire phenotypes responsible for its adaptation to different niches and hosts, focusing on virulence and AMR determinants.

Here, we first employed three different techniques to identify the five $K$. variicola strains isolated from different patients and sources of infection (Table 1): Vitek2 (BioMérieux), $16 \mathrm{~S}$ rRNA sequencing, and one-step multiplex PCR protocol (Fonseca et al., 2017). Vitek2 and 16S rRNA sequencing failed to distinguish $K$. variicola from $K$. pneumoniae strains. The difficulty in identifying $K$. variicola by Vitek 2 and $16 \mathrm{~S}$ rRNA sequencing has been pointed out previously by other authors (Rodríguez-Medina et al., 2019). In contrast, the multiplex PCR protocol identified the strains as $K$. variicola, which was later confirmed by sequencing the genome of all strains (Supplementary Table 2).

Based on the results of the antimicrobial susceptibility profile by disk diffusion, all strains were classified as MDR. MDR strains have been reported worldwide including a colistinresistant hypervirulent strain ( $\mathrm{Lu}$ et al., 2018). The biological assays showed that the strains presented different capacity 
TABLE 3 | The closest genome to each of Klebsiella variicola strains isolated in Brasilia, DF, Brazil, based on the absolute values of genome distances.

\begin{tabular}{|c|c|c|c|c|}
\hline Strain & Closest genome & Genetic distance & Institution & Geographic location \\
\hline Kv15 & GCF_003197065.1 & 0.006174 & Washington university & United States: St. Louis, Missouri \\
\hline Kv35 & GCF_003195165.1 & 0.006569 & Washington university & United States: St. Louis, Missouri \\
\hline Kv57 & GCF_900509865.1 & 0.004744 & Wellcome sanger institute & Belgium \\
\hline *Kv97 and Kv104 & GCF_003285185.1 & 0.006857 & Beijing institute of microbiology and epidemiology & China: Zhejiang \\
\hline
\end{tabular}

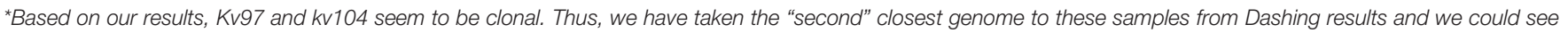
that the values were identical.

to survive and grow under incubation in blood and serum (Figures 1A,B). The strains Kv15, Kv35, and Kv97 presented growth profile in blood, as $K$. pneumoniae virulent strains described by Campos et al. (2018), reaching 1.5-2.5 × 109 CFUs after $60 \mathrm{~min}$ of incubation (Figure 1A). Regarding serum survival, all strains presented growth after $60 \mathrm{~min}$ of incubation (Figure 1B). Interestingly, Kv15, Kv35, and Kv57 showed ability to invade HEp-2 cells (Figure 1C), as previously observed in virulent $K$. pneumoniae (Campos et al., 2018). These three strains also showed significant higher values of CFUs recovery when compared to Kv97 and Kv104. As these two strains (Kv97 and Kv104) belong to the same clone (Table 1 and Supplementary Table 2), invasion assays showed different pathogenic potential between two groups: (i) one pathogenic group comprised of Kv15, K35, and Kv57 and other (ii), non-pathogenic formed by the clones Kv97 and Kv104. However, additional biological tests as murine infection is recommended to evaluate the virulence potential presented by the strains.

The capsular $(\mathrm{K})$ and lipopolysaccharide $(\mathrm{O})$ serotypes are useful methods for the rapid detection of outbreaks or high-risk clones since individual serotypes often vary in their virulence and invasive potential (Hsieh et al., 2012; Fang et al., 2016; Patro and Rathinavelan, 2019). In this study, we detected four different capsular synthesis loci (KL60, KL71, KL111, and KL20) and one lipopolysaccharide locus shared among the genomes (O3) (Table 2). In $K$. variicola, the prevalence of these serotypes is not yet elucidated and further studies are required. As expected, since they are clonal, the Kv97 and Kv104 strains presented the same $\mathrm{K}$ and $\mathrm{O}$ antigens, the same ST, and the same genomic content. However, the strains were isolated from different sources and patients: Kv94 from urine and Kv104 from blood, in the same period, January 2017 (Table 1). These results show the emergence of new K. variicola clones presenting capacity to colonize and cause infection in different tissues.

In this study, four new $K$. variicola STs (ST168, ST170, ST171, and ST172) have been identified. The investigation of genetic relationships among the MLST profiles deposited at the $K$. variicola database indicates that the $K$. variicola species is separated into two major CGs (Figure 2). Interestingly, this analysis have also divided the strains in two groups as the biological assays have. The pathogenic group (Kv15, Kv35, and Kv57) have been placed in the clonal CG that originates from the ST54, while the non-pathogenic group (Kv97 and Kv104) was placed in the other CG that seems to emerge from ST19 (Figure 2). This observation suggests that these CGs may represent different $K$. variicola populations with different phenotypes and different virulence and resistance dynamics as is known to happen in K. pneumoniae (Wyres et al., 2019, 2020). However, more studies are still necessary to better elucidate these differences. Furthermore, the diversity contrast among countries is also highlighted. In Bangladesh, for example, almost all (11/12) the sequenced strains belong to the same ST (ST 60) while in Brazil, almost every genome in the database belongs to a different ST which denotes the necessity of more sequencing efforts. Moreover, the distant genetic relationship among the five strains based on the MLST allelles together with their low ANI values, corroborates with the idea that the diversity of $K$. variicola in Brazil may be greater than what is currently known. In fact, the K. variicola MLST database is still new, and the majority of the STs are represented by only one genome. This suggests that the real magnitude of $K$. variicola diversity is yet to be seen and the number of sequenced genomes will be proportional to the contribution to unravel the epidemiology of this species.

Computational searches for ARGs detected those for betalactam $\left(b l a_{\mathrm{LEN}}\right)$, fosfomycin (fos $\left.\mathrm{A}\right)$, and quinolone (oqxAB). The beta-lactamase genes are one of the most prevalent in the species, being $b l_{\mathrm{LEN}}$ the most conserved (Potter et al., 2018). This high incidence of chromosomal $\beta$-lactamase LEN was expected since $K$. variicola is intrinsically resistant to ampicillin due to the presence of this gene (Rodríguez-Medina et al., 2019). In the same way, the oqxAB efflux pump is very conserved in the species as described in a recent study that detected the genes to be widespread among $K$. variicola strains isolated in United States (Potter et al., 2018). In contrast, despite being recently described by Potter et al. (2018) as a not very widespread gene, we identified in this study that virtually all the analyzed $K$. variicola genomes possess the fos A gene (Supplementary Material 2), a gene that is chromosomally carried in K. pneumoniae (Ito et al., 2017). Altogether these observations indicate that these genes are ubiquitous to the $K$. variicola species and suggest that $K$. variicola strains may be carriers of resistance markers worldwide.

$K$. variicola strains are frequently associated with different types of opportunistic infections. Recently, the first worldwide report of $\mathrm{Hmv} K$. variicola causing primary endodontic infection was described in Brazil (Nakamura-Silva et al., 2020). Furthermore, there are studies reporting the convergence of colistin resistance and hypervirulence in $K$. variicola in China (Lu et al., 2018) as well as multi-resistant hypervirulent 
strains in Bangladesh causing outbreaks with high mortality in newborns (Farzana et al., 2019). The studied strains exhibit genes and phenotypes related to virulence, mirroring factors considered classical in K. pneumoniae (Table 2 and Figure 1). These genes were shown to be very conserved among $K$. variicola genomes, except for the T6SS apparatus, and cannot explain the phenotypic differences observed between the strains (Figure 3). One remarkable observation was that although the $\mathrm{Hmv}$ phenotype was verified, the transcriptional regulator genes $r m p \mathrm{~A}$ and magA, considered pivotal in K. pneumoniae, were not found in the analyzed $K$. variicola strains. Our findings corroborate the results recently described by Nakamura-Silva et al. (2020) and also by Rodríguez-Medina et al. (2019) who did not find these genes in a $K$. variicola $\mathrm{Hmv}$ strain. Altogether, these observations corroborate the idea that $K$. variicola employs singular mechanisms of virulence not yet fully elucidated (Rodríguez-Medina et al., 2019).

Overall, our work shows the molecular and genomic characteristics of MDR $K$. variicola strains harboring different virulence factors belonging to novel clones. The results highlighting that $K$. variicola strains corroborating with the possibility that this species has its own virulome that still needs to be further elucidated (Rodríguez-Medina et al., 2019). Furthermore, their plastic genome can be responsible for the acquisitio of determinants that become a potential emerging pathogen.

\section{DATA AVAILABILITY STATEMENT}

The datasets generated in this study are available under the bioproject PRJNA 613075 (link: Klebsiella variicola (ID 613075)BioProject-NCBI (nih.gov)).

\section{ETHICS STATEMENT}

The studies involving human participants were reviewed and approved by the ethical approval was received from the School of Pharmaceutical Sciences of Ribeirão Preto, University of São Paulo, Brazil (approval no.: CEP/FCFRP 362; CAEE 36031914.9.0000.5403) and from the Faculdade de Medicina, Universidade de Brasília, Brasília, DF, Brazil (approval no. CEP/FMUnB 1.131.054; CAEE: 44867915.1.0000.558). The patients/participants provided their written informed consent to participate in this study.

\section{AUTHOR CONTRIBUTIONS}

AP-S and TC conceived and designed the experiments. AA and IS carried out biological tests related to bacterial strains isolation, biofilms, human blood and serum survival, bacterial invasion and toxicity to HEp-2 epithelial cell assays, and also statistical analysis. RN-S and MO-S conducted and analyzed the strains identification by sequencing the $16 \mathrm{~S}$ rRNA and onestep multiplex PCR, molecular typing and MLST, antimicrobial susceptibility, and hypermucosviscous tests. LC and NL carried out the genome sequencing. FA and GP performed genomic and bioinformatics analyzes. All authors contributed to writing this manuscript.

\section{FUNDING}

This work was supported by the Fundação de Amparo à Pesquisa do Estado de São Paulo (FAPESP) (grant no. 2013/225815). RN-S was a Ph.D. fellow of CAPES, therefore, this study was financed in part by the Coordenação de Aperfeiçoamento de Pessoal de Nível Superior (grant no 88882365161 and Finance Code 001).

\section{ACKNOWLEDGMENTS}

We are thankful to the team of curators from the Centro de Investigación Sobre Enfermedades Infecciosas (CISEI) in collaboration with the Centro de Ciencias Genómicas (CCGUNAM) (http://mlstkv.insp.mx/) for curating the data and making them publicly available in their database. We also thank Bruna Fuga, Fernanda Esposito, and Brenda Cardoso, from the Instituto de Ciências Biomédicas, Universidade de São Paulo, São Paulo, Brazil for extracting the genomic DNA and sequencing the bacterial strains. Finally, we also thank John Carpenter (Ribeirão Preto, SP, Brazil) for the English revision.

\section{SUPPLEMENTARY MATERIAL}

The Supplementary Material for this article can be found online at: https://www.frontiersin.org/articles/10.3389/fmicb. 2021.604031/full\#supplementary-material

Supplementary Figure 1 | Whole genome homology maps among the five genomes of this study, constructed with Mashmap. (A) Whole genome alignment between Kv97 and Kv104. (B) Whole genome alignment between Kv15 and Kv35. (C) Whole genome alignment between Kv15 and Kv57. (D) Whole genome alignment between Kv15 and Kv97.

Supplementary Figure 2 | Projection of the K. variicola genome distance matrix estimated by Dashing software into a two dimensional space using multidimensional scaling analysis. The clonal strains Kv97 and Kv104 were projected into the same space, thus only four of the five colored dots are visible.

Supplementary Table 1 | Genome assembly statistics for the five K. variicola strains analyzed.

Supplementary Material 1 | Antimicrobial susceptibilities of the five Klebsiella variicola strains and different antimicrobial agents tested.

Supplementary Material 2 | The Resfinder detection of ARGs in all the available $K$. variicola genomes. The first sheet summarizes the identification of fosA gene among the genomes. 


\section{REFERENCES}

Alcántar-Curiel, M. D., Blackburn, D., Saldaña, Z., Gayosso-Vásquez, C., Iovine, N., De La Cruz, M. A., et al. (2013). Multi-functional analysis of Klebsiella pneumoniae fimbrial types in adherence and biofilm formation. Virulence 4:2. doi: 10.4161/viru.22974

Baker, D. N., and Langmead, B. (2019). Dashing: fast and accurate genomic distances with HyperLogLog. Genome Biol. 20:265. doi: 10.1186/s13059-019$1875-1870$

Barbosa, V. A. A., and Lery, L. M. S. (2019). Insights into Klebsiella pneumoniae type VI secretion system transcriptional regulation. BMC Genomics 20:506 doi: 10.1186/s12864-019-5885-5889

Barrios-Camacho, H., Aguilar-Vera, A., Beltran-Rojel, M., Aguilar-Vera, E., Duran-Bedolla, J., Rodriguez-Medina, N., et al. (2019). Molecular epidemiology of Klebsiella variicola obtained from different sources. Sci. Rep. 9:10610. doi: 10.1038/s41598-019-46998-46999

Bortolaia, V., Kaas, R. S., Ruppe, E., Roberts, M. C., Schwarz, S., Cattoir, V., et al. (2020). ResFinder 4.0 for predictions of phenotypes from genotypes. J. Antimicrobial Chemotherapy 75, 3491-3500. doi: 10.1093/jac/dk aa345

Campos, T. A., Gonçalves, L. F., Magalhães, K. G., Martins, V. P., Pappas Júnior, G. J., Peirano, G., et al. (2018). A fatal bacteremia caused by Hypermucousviscous KPC-2 producing extensively drug-resistant K64-ST11 Klebsiella pneumoniae in Brazil. Front. Med. 5:265. doi:10.3389/fmed.2018. 00265

Carattoli, A., Zankari, E., Garciá-Fernández, A., Larsen, M. V., Lund, O., Villa, L., et al. (2014). In Silico detection and typing of plasmids using plasmidfinder and plasmid multilocus sequence typing. Antimicrobial Agents Chemotherapy 58, 3895-3903. doi: 10.1128/AAC.02412-14

Clinical and Laboratory Standards Institute (CLSI) (2020). Performance Standards for Antimicrobial Susceptibility Testing, M100, 30 Edn. Wayne, PA: Clinical and Laboratory Standards Institute (CLSI), M02-M07.

DeLeo, F. R., Kobayashi, S. D., Porter, A. R., Freedman, B., Dorward, D. W., Chen, L., et al. (2017). Survival of carbapenem-resistant Klebsiella pneumoniae sequence type 258 in human blood. Antimicrobial Agents Chemotherapy 61:e02533-16. doi: 10.1128/AAC.02533-2516

Fang, C. T., Shih, Y. J., Cheong, C. M., and Yi, W. C. (2016). Rapid and accurate determination of Lipopolysaccharide $\mathrm{O}$-antigen types in Klebsiella pneumoniae with a novel PCR-based O-genotyping method. J. Clin. Microbiol. 54, 666-675. doi: 10.1128/JCM.02494-15

Farzana, R., Jones, L. S., Rahman, M. A., Andrey, D. O., Sands, K., Portal, E., et al. (2019). Outbreak of hypervirulent multidrug-resistant Klebsiella variicola causing high mortality in neonates in Bangladesh. Clin. Infectious Dis. 68, 1225-1227. doi: 10.1093/cid/ciy778

Favre-Bonte, S., Joly, B., and Forestier, C. (1999). Consequences of reduction of Klebsiella pneumoniae capsule expression on interactions of this bacterium with epithelial cells. Infect. Immun. 67, 554-561.

Feldgarden, M., Brover, V., Haft, D. H., Prasad, A. B., Slotta, D. J., Tolstoy, I., et al. (2019). Validating the AMRFINder tool and resistance gene database by using antimicrobial resistance genotype-phenotype correlations in a collection of isolates. Antimicrobial Agents Chemotherapy 63:e0483-19. doi: 10.1128/AAC. 00483-419

Fonseca, E. L., Ramos, N., da, V., Andrade, B. G. N., Morais, L. L. C. S., Marin, M. F. A., et al. (2017). A one-step multiplex PCR to identify Klebsiella pneumoniae, Klebsiella variicola, and Klebsiella quasipneumoniae in the clinical routine. Diag. Microbiol. Infect. Dis. 87, 315-317. doi: 10.1016/j.diagmicrobio. 2017.01.005

Gautreau, G., Bazin, A., Gachet, M., Planel, R., Burlot, L., Dubois, M., et al. (2020). PPanGGOLiN: depicting microbial diversity via a partitioned pangenome graph. PLoS Computat. Biol. 16:e1007732. doi: 10.1371/journal.pcbi.100 7732

Ho, B. T., Dong, T. G., and Mekalanos, J. J. (2014). A view to a kill: the bacterial type VI secretion system. Cell Host Microbe 15, 9-21. doi: 10.1016/j.chom.2013. 11.008

Hsieh, P. F., Lin, T. L., Yang, F. L., Wu, M. C., Pan, Y. J., Wu, S. H., et al. (2012). Lipopolysaccharide O1 antigen contributes to the virulence in Klebsiella pneumoniae causing pyogenic liver abscess. PLoS One 7:e33155. doi: 10.1371/ journal.pone.0033155
Ito, R., Mustapha, M. M., Tomich, A. D., Callaghan, J. D., McElheny, C. L., Mettus, R. R., et al. (2017). Widespread fosfomycin resistance in Gram-negative bacteria attributable to the chromosomal fosA gene. mBio 8:e00749-17. doi: 10.1128/ mBio.00749-717

Jain, C., Koren, S., Dilthey, A., Phillippy, A. M., and Aluru, S. (2018a). A fast adaptive algorithm for computing whole-genome homology maps. Bioinformatics 34, i748-i756. doi:10.1093/bioinformatics/ bty597

Jain, C., Rodriguez-R, L. M., Phyllipy, A. M., Konstantinidis, K. T., and Aluru, S. (2018b). High throughput ANI analysis of $90 \mathrm{~K}$ prokaryotic genomes reveals clear species boundaries. Nat. Commun. 9:5114. doi: 10.1038/s41467-01807641-7649

Jia, B., Raphenya, A. R., Alcock, B., Waglechner, N., Guo, P., Tsang, K. K., et al. (2017). CARD 2017: expansion and model-centric curation of the comprehensive antibiotic resistance database. Nucleic Acids Res. 45, D566D573. doi: 10.1093/nar/gkw1004

Liu, B., Zheng, D., Jin, Q., Chen, L., and Yang, J. (2019). VFDB 2019: a comparative pathogenomic platform with an interactive web interface. Nucleic Acids Res. 47, D687-D692. doi: 10.1093/nar/gky1080

Lu, Y., Feng, Y., McNally, A., and Zong, Z. (2018). Occurrence of colistinresistant hypervirulent Klebsiella variicola. J. Antimicrobial Chemotherapy 73, 3001-3004. doi: 10.1093/jac/dky301

Maatallah, M., Vading, M., Kabir, M. H., Bakhrouf, A., Kalin, M., Nauclér, P., et al. (2014). Klebsiella variicola is a frequent cause of bloodstream infection in the Stockholm area, and associated with higher mortality compared to K. pneumoniae. PLoS One 9:e113539. doi:10.1371/journal.pone. 0113539

Magiorakos, A. P., Srinivasan, A., Carey, R. B., Carmeli, Y., Falagas, M. E., Giske, C. G., et al. (2012). Multidrug-resistant, extensively drug-resistant and pandrug-resistant bacteria: an international expert proposal for interim standard definitions for acquired resistance. Clin. Microbiol. Infect. 18, 268-281. doi: 10.1111/j.1469-0691.2011.03570.x

Mazloum, M., le Meur, M., Barnaud, G., and Messika, J. (2016). Hypermucoviscous Klebsiella pneumoniae pneumonia: follow the string! Intensive Care Med. 42, 2092-2093. doi: 10.1007/s00134-016-4363-y

Meier-Kolthoff, J. P., and Göker, M. (2019). TYGS is an automated highthroughput platform for state-of-the-art genome-based taxonomy. Nat. Commun. 10:2182. doi: 10.1038/s41467-019-10210-10213

Nakamura-Silva, R., Martins Domingues, de Macedo, L., Teixeira, L., Oliveira, da Silva, M., et al. (2020). First report of hypermucoviscous Klebsiella variicola subsp. variicola 2 causing primary endodontic infection. Clin. Microbiol. Infect. 27, 303-304.

Patro, L. P. P., and Rathinavelan, T. (2019). Targeting the sugary armor of Klebsiella species. Front. Cell Infect. Microbiol. 9:367. doi: 10.3389/fcimb.2019.00367

Piepenbrock, E., Higgins, P. G., Wille, J., Xanthopoulou, K., Zweigner, J., Jahn, P., et al. (2020). Klebsiella variicola causing nosocomial transmission among neonates - an emerging pathogen? J. Med. Microbiol. 69, 396-401. doi: 10.1099/ jmm.0.001143

Potter, R. F., Lainhart, W., Twentyman, J., Wallace, M. A., Wang, B., Burnham, C. A. D., et al. (2018). Population structure, antibiotic resistance, and uropathogenicity of Klebsiella variicola. mBio 9:e02481-18. doi: 10.1128/mBio. 02481-2418

Rodríguez-Medina, N., Barrios-Camacho, H., Duran-Bedolla, J., and GarzaRamos, U. (2019). Klebsiella variicola: an emerging pathogen in humans. Emerg. Microbes Infect. 8, 973-988. doi: 10.1080/22221751.2019.1634981

Seppey, M., Manni, M., and Zdobnov, E. M. (2019). BUSCO: assessing genome assembly and annotation completeness. Methods Mol. Biol. 1962, 227-245. doi: 10.1007/978-1-4939-9173-0_14

Srinivasan, V. B., and Rajamohan, G. (2020). Comparative genome analysis and characterization of a MDR Klebsiella variicola. Genomics 112, 3179-3190. doi: 10.1016/j.ygeno.2020.06.004

Stepanović, S., Ćirković, I., Ranin, L., and Švabić-Vlahović, M. (2004). Biofilm formation by Salmonella spp. and Listeria monocytogenes on plastic surface. Lett. Appl. Microbiol. 38, 428-432. doi: 10.1111/j.1472-765X.2004.01513.x

Swick, M. C., Morgan-Linnell, S. K., Carlson, K. M., and Zechiedrich, L. (2011). Expression of multidrug efflux pump genes acrAB-tolC, mdfA, and norE in Escherichia coli clinical isolates as a function of fluoroquinolone and multidrug resistance. Antimicrobial Agents Chemotherapy 55, 921-924. doi: 10.1128/AAC 00996-910 
Weisburg, W. G., Barns, S. M., Pelletier, D. A., and Lane, D. J. (1991). 6 S ribosomal DNA amplification for phylogenetic study. J Bacteriol. 173, 697-703.

Wick, R. R., Heinz, E., Holt, K. E., and Wyres, K. L. (2018). Kaptive web: user-friendly capsule and lipopolysaccharide serotype prediction for Klebsiella genomes. J. Clin. Microbiol. 56:e00197-18. doi: 10.1128/JCM.00197-118

Wick R. R., Judd L. M., Gorrie C. L., and Holt K. E. (2017). Unicycler: resolving bacterial genome assemblies from short and long sequencing reads. PLOS Comput. Biol. 13:e1005595. doi: 10.1371/journal.pcbi.1005595

Wilksch, J. J., Yang, J., Clements, A., Gabbe, J. L., Short, K. R., Cao, H., et al. (2011). MrKH, a novel c-di-GMP-dependent transcriptional activator, controls klebsiella pneumoniae biofilm formation by regulating type 3 fimbriae expression. PLoS Pathogens 7:e1002204. doi: 10.1371/journal.ppat. 1002204

Wyres, K. L., Lam, M. M. C., and Holt, K. E. (2020). Population genomics of Klebsiella pneumoniae. Nat. Rev. Microbiol. 18, 344-359. doi: 10.1038/s41579019-0315-311

Wyres, K. L., Wick, R. R., Judd, L. M., Froumine, R., Tokolyi, A., Gorrie, C. L., et al. (2019). Distinct evolutionary dynamics of horizontal gene transfer in drug resistant and virulent clones of Klebsiella pneumoniae. PLoS Genetics 15:e1008114. doi:10.1371/journal.pgen. 1008114

Zhou, Z., Alikhan, N. F., Sergeant, M. J., Luhmann, N., Vaz, C., Francisco, A. P., et al. (2018). Grapetree: visualization of core genomic relationships among 100,000 bacterial pathogens. Genome Res. 28, 1395-1404. doi: 10.1101/gr. 232397.117

Conflict of Interest: The authors declare that the research was conducted in the absence of any commercial or financial relationships that could be construed as a potential conflict of interest.

Copyright (C) 2021 Campos, Almeida, Almeida, Nakamura-Silva, Oliveira-Silva, Sousa, Cerdeira, Lincopan, Pappas and Pitondo-Silva. This is an open-access article distributed under the terms of the Creative Commons Attribution License (CC BY). The use, distribution or reproduction in other forums is permitted, provided the original author(s) and the copyright owner(s) are credited and that the original publication in this journal is cited, in accordance with accepted academic practice. No use, distribution or reproduction is permitted which does not comply with these terms. 